\title{
The impact of treatment, socio-demographic and clinical characteristics on health-related quality of life among Hodgkin's and non-Hodgkin's lymphoma survivors: a systematic review
}

\author{
Simone Oerlemans • Floortje Mols • Marten R. Nijziel • \\ Marnix Lybeert • Lonneke V. van de Poll-Franse
}

Received: 10 February 2011 / Accepted: 5 June 2011 / Published online: 14 June 2011

(C) The Author(s) 2011. This article is published with open access at Springerlink.com

\begin{abstract}
Cancer survivors are at risk of experiencing adverse physical and psychosocial effects of their cancer and its treatment. Both Hodgkin's lymphoma (HL) and nonHodgkin's lymphoma (NHL) survivors face problems that can affect their health-related quality of life (HRQoL). The authors systematically reviewed the literature on HRQoL among HL and NHL survivors. A PubMed and PsychINFO literature search for original articles published until May 2011 was performed. Twenty-four articles, which met the predefined inclusion criteria, were subjected to a quality checklist. HL survivors showed the most problems in (role) physical, social and cognitive functioning, general health, fatigue and financial problems. In addition, HL survivors treated with a combination of therapies, with older age and female sex reported worse HRQoL. NHL survivors showed the most problems in physical functioning, appetite loss, vitality and financial problems. Having had chemotherapy was negatively
\end{abstract}

This manuscript is not being considered for publication elsewhere, and the findings of this manuscript have not been previously published.

S. Oerlemans $(\varangle) \cdot$ F. Mols $\cdot$ L. V. van de Poll-Franse

Research Department, Comprehensive Cancer Centre South,

PO Box 231, 5600 AE Eindhoven, The Netherlands

e-mail: s.oerlemans@ikz.nl

S. Oerlemans $\cdot$ F. Mols $\cdot$ L. V. van de Poll-Franse

Center of Research on Psychology in Somatic Diseases (CoRPS),

Tilburg University,

Tilburg, The Netherlands

\section{R. Nijziel}

Internal Medicine/Haemato-Oncology, Máxima Medical Centre, Eindhoven, The Netherlands

M. Lybeert

Department of Radiation Oncology, Catharina Hospital,

Eindhoven, The Netherlands associated with HRQoL, but no differences in chemotherapy regimens were found. Furthermore, in NHL survivors not meeting public exercise guidelines, HRQoL is low but can be improved with more exercise. More research on the longitudinal comparison between HL and NHL survivors and healthy controls should be performed in order to better understand the long-term (side) effects of treatment on HRQoL and possibilities to alleviate these.

Keywords Quality of life · Hodgkin's lymphoma · NonHodgkin's lymphoma . Cancer treatment . Cancer survivors

\section{Introduction}

Treatment of cancer has improved considerably in the past decades resulting in more (long-term) survivors. A person diagnosed with cancer is defined a survivor from the moment of diagnosis through the balance of his or her life [1]. The number of cancer survivors in the United States (US) has increased steadily and is currently estimated to be 11.1 million [2]. The number of lymphoma survivors has relatively increased even more. On January 1, 2008, there were approximately 167,000 Hodgkin's lymphoma (HL) survivors and approximately 454,000 non-Hodgkin's lymphoma (NHL) survivors in the US [2]. In the Nordic European Countries (NEC: Denmark, Faroe Islands, Finland, Iceland, Norway, Sweden), there were approximately $10,500 \mathrm{HL}$ survivors and approximately 31,500 NHL survivors at the end of 2007 [3].

Although there are similarities between these subtypes of lymphoma, the incidence and age of onset are quite different. The annual incidence of HL is one in 35,000 in the US [2] and one in 47,000 in the NEC [3], with approximately 8,500 new 
cases in the US [2] and 558 new cases in the NEC [3] annually. Onset occurs most frequently between the ages of 20 and 35 years. Between 35 and 50 years, it occurs less often especially in females, but from the age of 50 onward there is again a rise in incidence with age [2]. The lifetime prevalence of $\mathrm{HL}$ is one in 430 [2]. With respect to NHL, the annual incidence is one in 5,000, with approximately 65,000 new cases in the US [2] and 73,000 new cases in the European Union (NEC numbers are unavailable) [4]. The disease occurs predominantly in individuals aged over 45 years and the lifetime prevalence of NHL is one in 50 [2].

Due to chemotherapy, radiotherapy and stem cell transplantation, the survival of these patients has improved dramatically in the seventies and eighties, but has nowadays levelled off. In effect, most trials focus on maintaining the high level of cure, while reducing the long-term effects of treatment. To date, more than $80 \%$ of patients diagnosed with HL are expected to live free of disease for 5 years or more after diagnosis [5]. The overall 5-year survival rate for all types of NHL (1999-2005) is 50-60\%. The statistics vary depending on the cell type, stage of disease at diagnosis, treatment and age of the patient [5].

As cancer survivors are living longer, they are at risk of experiencing adverse physical and psychosocial long-term effects of the fact that they had cancer or of their treatment [6-9]. The long-term HL and NHL survivors, especially, face specific problems, concerning mainly chronic medical as well as psychosocial complications that can affect their health-related quality of life (HRQoL). Fatigue, depression, marital disruption and problems with infertility are frequently reported concerns by these survivors, not to mention problems with insurances and mortgages [6, 8-11].

Only recently, the focus of published papers has shifted from improvement of survival to HRQoL. In December 2009, a review concerning HRQoL in lymphoma survivors has been published [12]. This review described the HRQoL of both HL and NHL survivors combined, which may delude conclusions as differences in age of onset, treatment and overall survival time between HL and NHL greatly influences HRQoL. In addition, four prospective and two cross-sectional studies, all published between 2004 and 2009, were not included in this review and especially these prospective studies contain important information. Furthermore, and most important, the review did not provide information about the clinical implications of its findings. Many studies base their conclusions on statistical significance, but clinical significance should also be studied for the representation of clinically important differences to patients. Our review will therefore distinguish itself by a separate discussion of both types of lymphoma, a more complete and updated overview of studies, and by providing information about clinical significance of the findings. The aim of this systematic review was (1) to evaluate the quality of the included studies, (2) to identify the HRQoL domains and symptoms that are clinically relevant affected in HL and NHL survivors, (3) to evaluate the relation between treatment and HRQoL and (4) to evaluate potential differences in socio-demographic and clinical characteristics.

\section{Methods}

\section{Search strategy}

The electronic databases of PubMed and PsychINFO were searched to find all articles up to May 2011 using the terms 'Hodgkin's' and 'non-Hodgkin's lymphoma' in combination with survivors, long-term, (health-related) quality of life, and HRQoL. The reference lists of all publications were checked to retrieve additional publications.

\section{Selection criteria}

Studies in English on HRQoL in HL and NHL adult survivors were included if they used a multidimensional HRQoL questionnaire. Studies that merely focused on onedimensional aspects of HRQoL such as fatigue, anxiety or depression were excluded from this review because this is not consistent with the multidimensional concept of HRQoL (i.e. the existential influence of disease on physical, emotional and social functioning). Also, studies that involved a variety of tumours including HL or NHL, focused on adult survivors of childhood cancer, and studies not published in peer-reviewed journals were excluded. Furthermore, the focus of the study had to be either one or more of the following: (1) comparison with a normative population, (2) studying the relation between treatment and HRQoL, (3) studying the relation between sociodemographic or clinical characteristics and HRQoL.

The search resulted in 270 hits. Based on titles and abstracts, 24 articles met our selection criteria and were included in this review (Fig. 1).

\section{Quality assessment}

The methodological quality of the selected studies was assessed using a 12-item standardized checklist of predefined criteria which was a modified version of an established criteria list for systematic reviews (Table 1) [13, 14]. Each item of a study, which met our criteria, was assigned one point. If an item that did not meet our criteria was described insufficiently, or not at all, then zero points were assigned. The highest possible score was 12 . Studies scoring $\geq 8$ points were considered to be of 'high quality'. Studies scoring $<6$ points or $6-8$ points were rated respectively as low and moderate quality. 
Fig. 1 Flow diagram of papers accepted and rejected during selection procedure

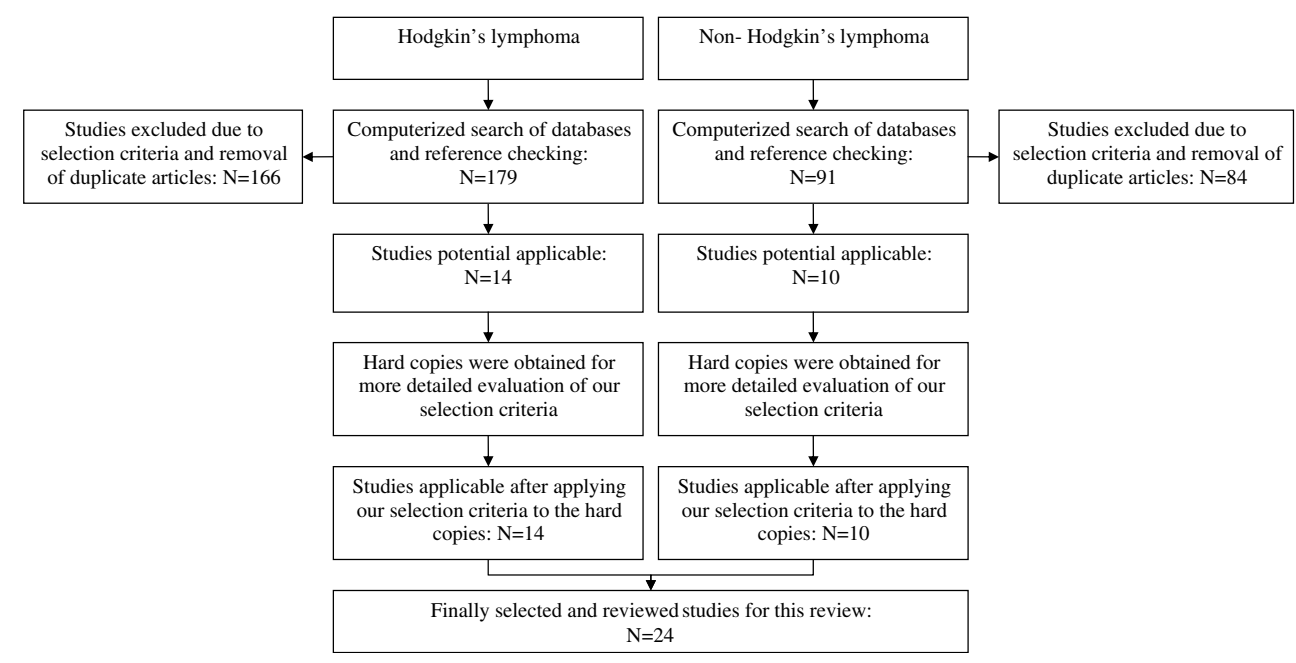

Criteria for clinically important difference

The following criteria were used to determine clinically important differences. For the European Organization for

Table 1 List of criteria for assessing the methodological quality of studies on health-related quality of life among Hodgkin's and nonHodgkin's lymphoma

Positive if with respect to

Quality of life assessment

1. A validated (Health-Related) Quality of Life Questionnaire is used (e.g. SF36, EORTC-C30)

Study population

2. A description of at least two socio-demographic variables is included

3. A description of at least two clinical variables of the described patient population (e.g. tumour stage at diagnosis) is present

4. Inclusion and/or exclusion criteria are described

5. Participation rates for patient groups are described and are more than $60 \%$

6. Information is given about the degree of selection of sample (information is given about the ratio respondents vs. nonrespondents)

Study design

7. The study size consists of at least 50 participants (arbitrarily chosen)

8. The data are prospectively gathered

9. The process of data collection is described (e.g. interview or selfreport)

Results

10. The results are compared between two groups or more (e.g. healthy population, groups with different treatment or age) and/or results are compared with at least two time points (e.g., longitudinally vs. post-treatment)

11. Mean, median, standard deviations or percentages are reported for the most important outcome measures

12. Statistical proof for the findings is reported
Research and Treatment of Cancer Quality of Life Questionnaire (EORTC QLQ-C30), a score greater than or equal to a 10-point difference on subscales reflects a clinical important difference [9, 15-18]. Concerning the RAND Short Form-36 (SF-36), differences of $\geq 2$ points for role physical functioning; $\geq 3$ points for physical functioning, social functioning, bodily pain, general health, vitality, mental health and the component scales; $\geq 4$ points for role emotional functioning are considered clinically meaningful $[19,20]$. For the other questionnaires and some subscales, Norman's 'rule of thumb' was used whereby a $\approx 0.5 \mathrm{SD}$ difference indicates a threshold of discriminating change in HRQoL scores of a chronic illness [21].

\section{Results}

\section{Study characteristics}

In total, 24 studies were included (14 HL $[9,15,16,22-$ 32]) and ten NHL [11, 17, 33-40]) all published between February 1994 and November 2010. Only one study was conducted outside the US and Europe [40]. The time since diagnosis ranged between circa 2 months and 44 years. The most frequently used questionnaires of HRQoL were the EORTC QLQ-C30 (ten studies) [41] and the SF-36 (11 studies) [42]. Two studies used the Schedule for the Evaluation of Individual Quality of Life-Direct Weighting (SEIQoL-DW) [43], and three studies used the Functional Assessment of Cancer Therapy-General (FACT-G) [44].

With respect to HL, two studies had a prospective design, and 12 studies had a cross-sectional design. Of the 14 studies, ten cross-sectional studies compared HL survivors and the general population, two prospective and eight cross-sectional studies evaluated the relation with treatment, and two prospective and nine cross-sectional 
studies reported about potential differences in sociodemographic and clinical characteristics (Table 2). With respect to NHL, four studies had a prospective design and six studies had a cross-sectional design. Of the ten studies, two prospective and three cross-sectional studies compared NHL survivors and the general population, three prospective and two cross-sectional studies evaluated the relation with treatment, and nine studies reported about the potential differences in socio-demographic and clinical characteristics (Table 3).

The evaluation of the methodological quality of the studies by the reviewers (SO, FM, LP) yielded the following results. On items $1,5,7,8,9,11$ and 12 there was disagreement once, and on items 2, 3 and 10 there was no disagreement. On items 4 and 6 (see Table 1), there was disagreement a couple of times, mostly due to differences in interpretation of the text. These were solved through discussion in a consensus meeting. The methodological quality of all included studies ranged from 8 to 12 points and was thus considered to be of high quality. General shortcomings concerned mainly the lack of information on non-respondents $(n=11)$ and the lack of a prospective design $(n=16)$.

\section{Hodgkin's lymphoma}

\section{HRQoL domains: HL survivors vs. normative samples}

Four cross-sectional studies found clinically important lower physical functioning scores for survivors compared to a normative population $[9,26,30,32]$. No clinically important differences were found in six studies [15, 22, 24, $27,28,31]$.

Five studies found that HL survivors had clinically important lower scores on social functioning compared to normative samples $[9,15,22,24,26]$. Three studies found no clinically important differences on social functioning $[27,28,30]$.

One study among 98 survivors that survived more than 8 years found that HL survivors had clinically important lower scores on emotional functioning compared to the normative sample [22] while seven studies found no clinically important differences [9, 15, 24, 26-28, 30].

Five studies found that HL survivors had clinically important lower scores on role physical functioning compared to the normative sample $[9,22,26,27,30]$. Three studies found no clinically important differences [15, 24, 28].

No clinically important differences were found between HL survivors and normative samples with respect to Global health state (six studies) [15, 22, 24, 27, 28, 31].

Three cross-sectional studies found that HL survivors had clinically important lower scores on general health compared to the normative sample $[9,26,30]$. Two cross- sectional studies found no clinically important differences on general health $[28,31]$.

No clinically important differences were found between HL survivors and normative samples regarding mental health scores (four studies) [9, 26, 28, 30].

\section{Symptoms: HL survivors vs. normative samples}

Clinically important worse scores of HL survivors were reported on several symptoms; cognitive problems [15, 22, 27], financial difficulties [15, 22, 24], fatigue/vitality [9, 15, 22], dyspnea [22, 27] and insomnia [15, 22] were reported most often. Diarrhoea [22] and pain [26] were reported by one study each. Three studies found no clinically important differences between HL survivors and normative samples $[9,13,30]$.

\section{Treatment and HRQoL in HL survivors}

One prospective study among 247 early stage HL survivors [23] found clinically important lower scores on vitality among patients treated with a combination of radiotherapy and chemotherapy compared to patients treated with radiotherapy alone, but only in the first year after treatment. This effect was also found in a cross-sectional study among $126 \mathrm{HL}$ survivors in Austria [25]. They reported that patients treated with a combination of radiotherapy and chemotherapy showed clinically important lower scores on physical functioning and clinically important higher scores on pain, fatigue and dyspnoea compared to patients who were treated with radiotherapy or chemotherapy alone. Two small cross-sectional studies also found clinical important higher scores on dyspnoea. One found that 26 patients treated with mantle field irradiation reported higher scores compared with patients treated without $(n=16)$ [29]. The other study found that 37 patients treated with high dose chemotherapy and stem cell transplantation reported higher scores compared with patients treated with conventional chemotherapy $(n=61)$ [22]. Another prospective and four cross-sectional studies found no effect of treatment on HRQoL [9, 16, 24, 28, 32].

\section{Socio-demographic and clinical characteristics in $H L$ survivors}

Three cross-sectional and one prospective study observed that older patients reported clinically important worse outcomes [9, 16, 24, 27]. Six studies reported contradicting differences in HRQoL according to gender [9, 15, 16, 24, 27, 29], three studies found clinically important worse scores for women $[9,24,27]$, one found only statistically worse scores for women [16], one found worse scores for men [29] and one found no differences [15]. Two cross- 


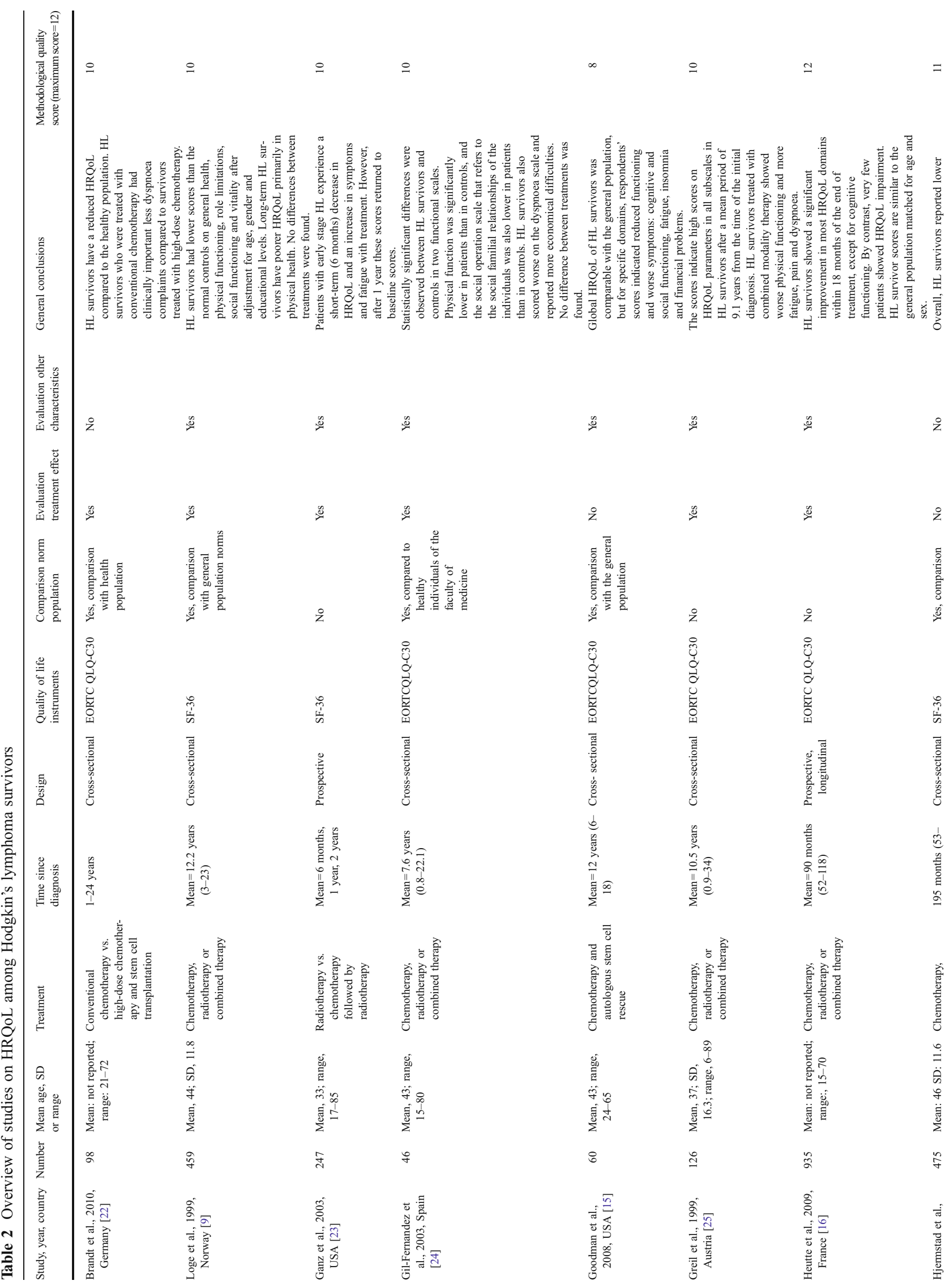




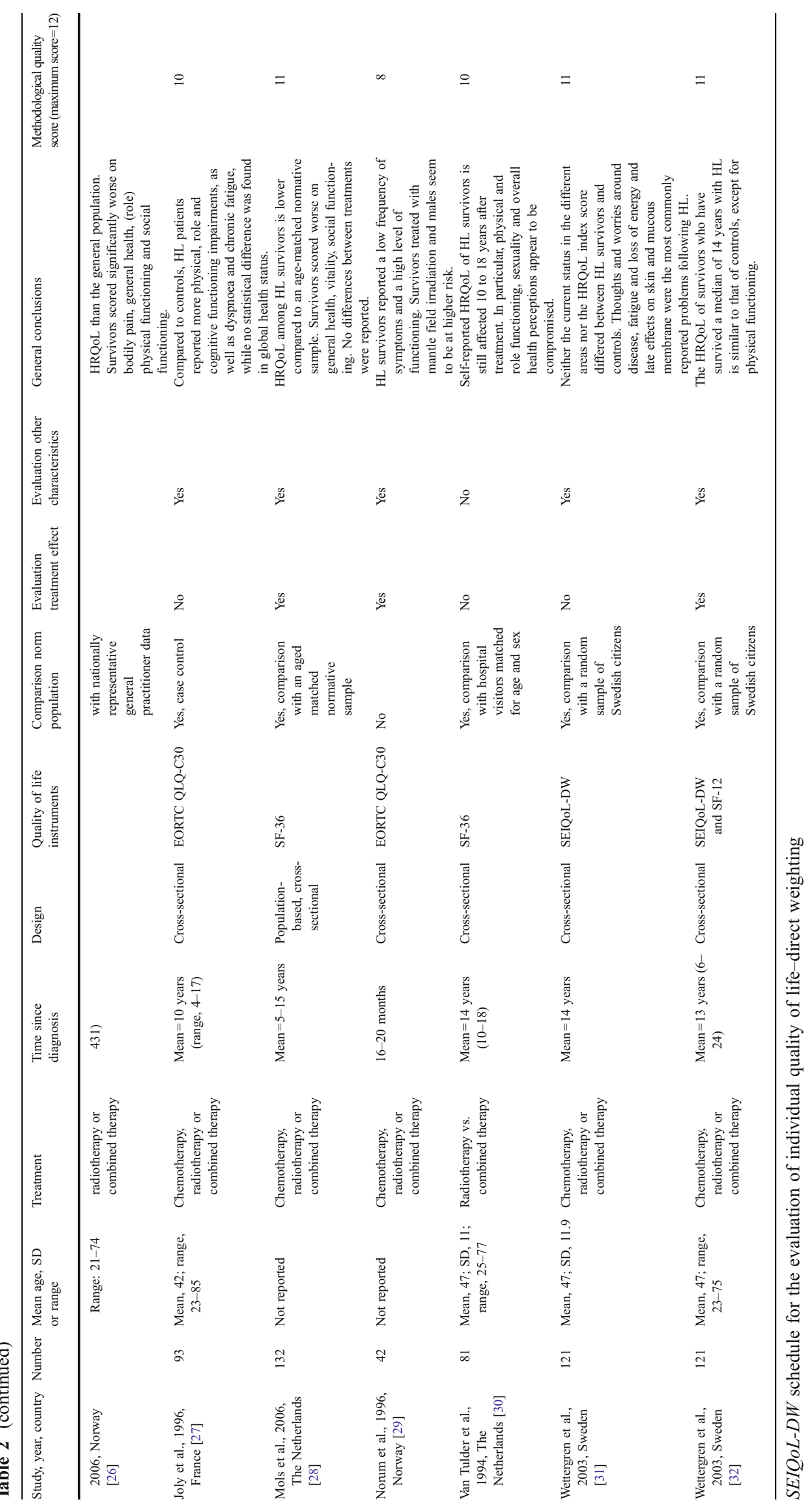




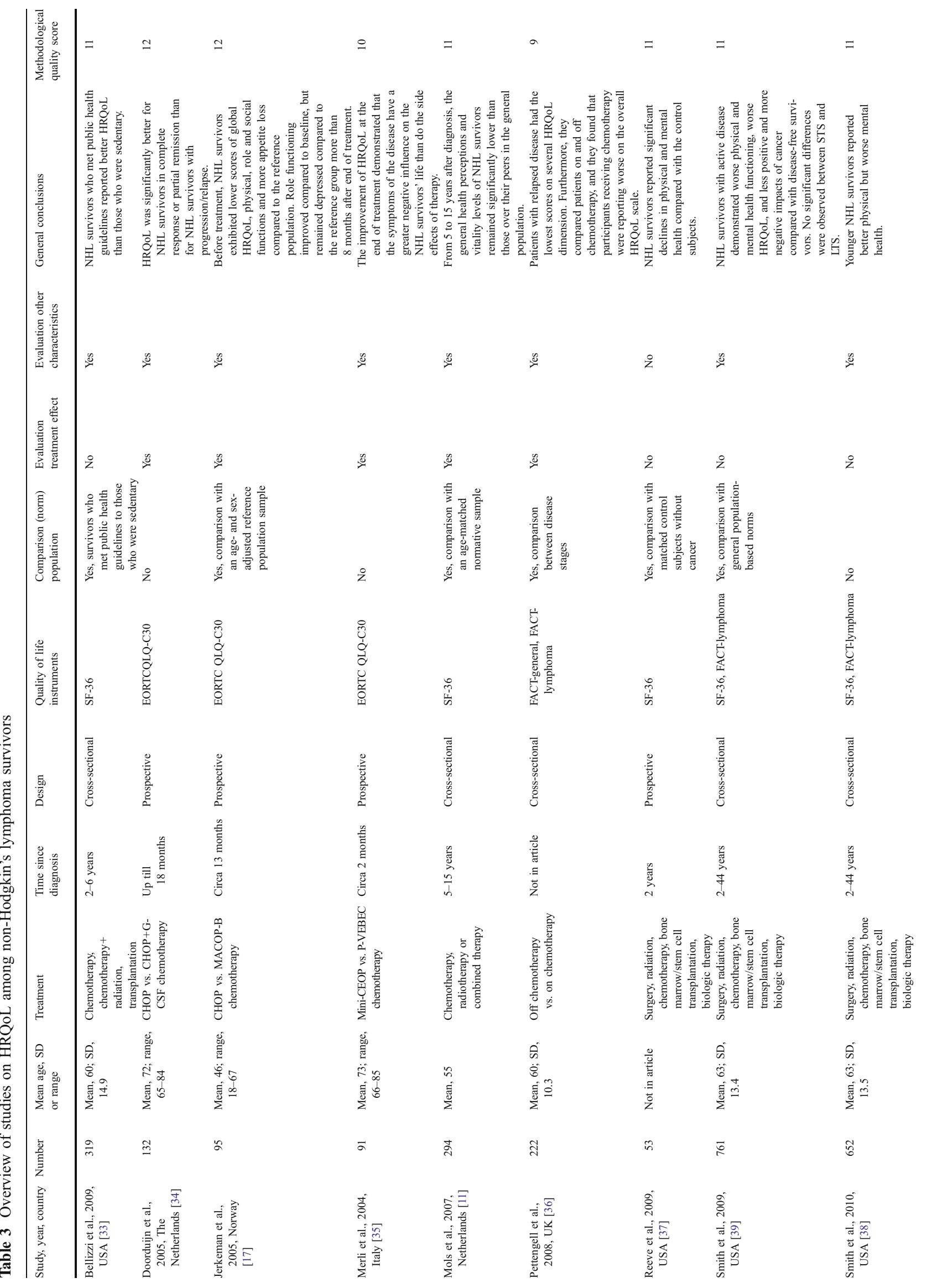


sectional studies reported that more advanced disease stage or recurrences were associated with reduced HRQoL; however, no information about clinically important differences could be obtained [15, 32]. One study found remarkably that patients with stage IB-IIB scored significantly and clinically important lower on physical functioning and physical role limitations compared to patients with stage IA-IIA, IIIA-IVA and IIIB-IVB [9].

The impact of length of survival on HRQoL was reported in a cross-sectional study, showing that patients who had survived 10-15 years after diagnosis reported clinically important higher HRQoL scores than patients who had survived 5-9 years [28].

Non-Hodgkin's lymphoma

HRQoL domains: NHL survivors vs. normative samples

In a prospective study among 95 Norwegians [17], patients showed clinically important lower scores on physical functioning up until 5 months after start of therapy compared to the normative sample. However, 8 months after end of treatment, the difference was no longer clinically relevant. A Dutch cross-sectional study among 294 survivors [11] and an American cross-sectional study of 319 survivors found no clinically important lower scores for survivors compared to the normative sample on physical functioning [33]. A cross-sectional study of 761 survivors [39] showed clinically important lower scores on the physical component scale. Another prospective study found statistically lower scores 2 years post-diagnosis; however, no information about clinically important differences could be obtained [37].

A prospective study [17] exhibited clinically important lower scores on social functioning up until 6 months after start of therapy compared to the reference population. However, 8 months after end of treatment, the difference was no longer clinically relevant. Two years post-diagnosis, another prospective study found statistically lower scores; however, no information about clinically important differences could be obtained [37]. A cross-sectional study found no clinically important differences with respect to social functioning [11].

A prospective study [17] showed clinically important lower scores on role physical function compared to the general population, and these scores remained clinically important lower until the end of the study ( 8 months). Another prospective study found statistically lower scores 2 years post-diagnosis; however, no information about clinically important differences could be obtained [37]. However, a cross-sectional study found no clinically important differences regarding role physical function [11]. 
Two prospective and a cross-sectional study did not find statistically or clinically important differences with respect to emotional functioning between NHL survivors and the normative populations [11, 17, 37].

A prospective study [17] exhibited clinically important lower scores on global health state up until 5 months after start of therapy compared to the reference population. However, 8 months after the end of treatment, the difference was no longer clinically relevant.

In addition, a cross-sectional study among 294 survivors showed clinically important lower scores on general health [11]. A prospective study found statistically lower scores 2 years post-diagnosis; however, no information about clinically important differences could be obtained [37]. Three cross-sectional studies did not find clinically important differences between NHL survivors and the reference population on mental health [11, 33, 39].

\section{Symptoms: NHL survivors vs. normative samples}

A prospective study [17] showed clinically important lower scores on appetite loss, constipation, fatigue and dyspnoea up until respectively 3 months for the first two symptoms and 5 months for the last two symptoms after start of therapy compared to the reference population. However, 8 months after the end of treatment the difference was no longer clinically relevant. Furthermore, they found that 9 months after the end of treatment until the end of the study, survivors had clinically more financial difficulties than the normative sample. A cross-sectional and prospective study found statistically lower scores for survivors on vitality [28,37], clinically important differences were only found by the cross-sectional study [28].

\section{Treatment and HRQoL in NHL survivors}

Three prospective studies showed no significantly different outcomes regarding HRQoL between patients treated with different chemotherapy regimens $[17,34,35]$. Two crosssectional studies found that, compared to patients who did not receive chemotherapy, patients who did receive chemotherapy experienced clinically important worse overall health functioning [36] and social well-being [11].

\section{Socio-demographic and clinical characteristics in NHL survivors}

A prospective study did not find a relation between age and HRQoL outcome [17]. One cross-sectional study [11] found that older patients scored significantly lower on physical functioning than younger patients; however, no information about clinically important differences could be obtained. Another cross-sectional study found clinical meaningful worse physical HRQoL scores for survivors who were older at study enrolment [38].

Two prospective studies [34, 35] found that survivors with progressive disease had clinically meaningful lower HRQoL than patients who were free of disease. Another prospective study found no relation between disease stage and HRQoL [17]. Two cross-sectional studies found statistically lower HRQoL scores for survivors with active (relapsed) disease compared to disease-free survivors [36, 39]; clinically important differences were found in one of them [36].

The impact of length of survival was reported in a crosssectional study [11] showing that patients who had survived 10-15 years after diagnosis reported clinically important higher HRQoL scores than patients who had survived 59 years, but this was not observed by another study [39] that compared short-term (2-5 years after diagnosis) and long-term ( $\geq 5$ years after diagnosis) survivors.

Two prospective studies [34, 35] investigated HRQoL in elderly patients in relation to the age-adjusted International Prognostic Index which comprises three factors (performance status, lactate dehydrogenase and stage) [45]. These studies found that patients with a low age-adjusted International Prognostic Index had a clinically meaningful better HRQoL than patients with a high age-adjusted International Prognostic Index. One prospective study found no relation between International Prognostic Index and HRQoL [17].

Two cross-sectional studies found that survivors meeting public health exercise guidelines reported a clinically meaningfully better mental and physical health [33, 40] than survivors not meeting these guidelines. Even more important, one of these studies [33] found that there was a significant dose-response pattern in which more exercise resulted in better mental and physical health.

\section{Discussion}

This systematic review summarized and evaluated the results of studies focusing on the HRQoL of $\mathrm{HL}$ and NHL survivors. It is a remarkable fact that the majority of these studies concerned HL and not NHL, certainly in view of the number of patients being treated $(8,500$ vs. 65,000$)$ or the number of survivors $(165,000$ vs. 440,000) [5]. Another point is that the first included study on HRQoL in HL was published in 1994, whereas all included studies on HRQoL in NHL were published after 2004.

The quality scores of the included HL studies ranged from eight to 12 points, which indicate a high methodological quality. The shortcomings of these studies were mainly the lack of a prospective design and lack of information on non-respondents. The HRQoL domains that 
were affected the most in these patients and represent clinically important differences to patients were (role) physical, social and cognitive functioning, general health, fatigue and financial problems, and fewer dyspnoea and insomnia. Clinically important differences in emotional functioning, diarrhoea and pain were reported once. No clinically important differences were found in the included studies for physical functioning and mental health. Based on the studies included in this review, HL survivors who received a combination of chemotherapy and radiotherapy $[23,25]$ had worse scores on HRQoL domains. A clinically important higher score on dyspnoea was found by all therapies, and this suggests that treatment in general results in problems [29]. However, most of the studies found no differences. In addition, HL survivors with older age and females reported worse outcomes. As expected, patients with a longer survival time reported better outcomes compared to those more recently diagnosed.

With respect to the included NHL studies, it was not possible to divide the results section in aggressive and indolent lymphomas due to lack of information within most available studies. The quality scores of NHL studies ranged from nine to 12 points, which indicate a high methodology quality. The shortcomings were mainly a lack of a prospective design. The HRQoL domains that were affected the most in NHL survivors and represent clinically important differences to patients were physical functioning, appetite loss, vitality and financial problems. Clinically important differences in social functioning, role physical functioning and global health were mentioned once up until 5 months after treatment but waned over time. When comparing different chemotherapy regimens, no differences were reported. Nevertheless, having had chemotherapy was associated with clinically important lower scores on social well-being [11] and overall health functioning [36] as reported by two cross-sectional studies in (long-term) cancer survivors. Interestingly, the effect of exercise was studied in NHL patients, whereas this has not been investigated in HL survivors. NHL survivors that met public health exercise guidelines reported a clinically important better HRQoL than survivors that did not meet exercise guidelines [33, 40], and even more important, more exercise resulted in a better mental and physical health [33]. Most studies showed worse HRQoL for survivors with aggressive disease or partial response, no response or progressive disease [34-36, 39], and those with a high age-adjusted Prognostic Index [34, 35], which is well understandable.

The criterion of clinically important differences is very important to specify those domains of HRQoL that are affected in survivors. Most of the included studies based their conclusions only on statistical significance. Sometimes differences between survivors and comparative groups were statistically significant, but not clinically important for patients. Therefore, researchers should always use a criterion for the interpretation of clinical relevance instead of only evaluating the statistical significance to really attribute to the care of patients. Of the 17 included studies that compared HRQoL between survivors and a normative population, only seven $[9,15-17,28,32,39]$ studies used a criterion to determine clinically relevant differences.

When comparing different studies, certainly in the field of HRQoL, there are many limitations. Seventeen of 22 included papers had a cross-sectional design $[9,11,15,24-33,39,40]$. A limitation of this methodology is that it is not possible to draw causal relationships. In addition, these studies may have survivorship bias because patients that do relatively worse will not participate as they are too ill or dead. A prospective design study provides better relevant answers about causality, for example the temporal direction between treatment and HRQoL, but only five studies had this design. Also, the lack of information in some studies on non-respondents or possible bias makes it more difficult to determine the trustworthiness of a study. Future studies should therefore always try to collect data on non-respondents or discuss the possible risk of bias. Although there are inherent relationships between HRQoL dimensions, we discussed the dimensions separately to identify which specific dimensions are most affected. This does not mean that the unmentioned dimensions could not be affected. However, the underlying mechanisms between the relations in HRQoL dimensions and symptoms are understudied and not yet clear. Therefore, studies focusing on symptom clustering are needed.

The different HRQoL questionnaires used, predominantly the EORTC QLQ-C30 (disease-specific questionnaire) and the SF-36 (generic questionnaire) made it difficult to compare results, as the various scales do not exactly measure the same HRQoL dimensions. The questionnaires in the included studies were almost all generic or disease specific. Generic questionnaires are designed to measure health in general, and are therefore appropriate for a wide range of patient groups and also the general population, but are less sensitive to detect certain aspects of disease and treatment that are relevant to a specific patient group. The EORTC QLQ-C30 is a disease specific questionnaire, but consists of such questions that this questionnaire is also applicable to the general population [46]. In addition to these generic and disease-specific questionnaires, lymphoma-specific questionnaires should be used to detect, with more sensibility, side effects and symptoms particularly relevant to HL and NHL survivors. However, cancer-specific questionnaires are relatively new and underdeveloped and therefore used infrequently. Only two studies $[36,39]$ used a lymphoma-specific questionnaire, the FACTlymphoma, which was developed in 2004 [44].

The American Cancer Society defines 'long-term survivors' as every person who is still alive 5 years after diagnosis [47]. Six studies, five HL [15, 28, 30-32] and one NHL [11], focused on patients who had survived more than 
5 years. Only one recent study focused on the longitudinal HRQoL of HL survivors [16]. Especially these kinds of studies are important in view of the growing number of survivors to identify as soon as possible negative long-term effects, certainly when taken into consideration the implementation of new treatments.

If we compare the results of the 11 cross-sectional and two prospective studies among the HL survivors, some crosssectional studies are consistent with the prospective studies on points as comparison with the norm population, relation with received treatment, and socio-demographic and clinical differences. However, if we compare the two prospective HL studies, one [23] did find a relation with treatment while the other [16] did not. With respect to NHL studies, again some of the crosssectional studies (four) are consistent with the prospective ones. However, if we compare the three prospective studies, only consistent results concerning the absence of the relation with treatment on HRQoL were found [17, 34, 35].

In conclusion, the reviewed literature about the HRQoL of HL and NHL survivors reflects that several domains, even in long-term survivors, are affected. Overall, HL survivors experience the most problems in (role) physical, social and cognitive functioning, general health, fatigue and financial problems. In addition, HL survivors with older age and female sex reported worse outcomes. NHL survivors experience the most problems in physical functioning, appetite loss, vitality and financial problems. However, these results are less clear as only a limited number of studies are performed among NHL survivors. Furthermore, importantly the HRQoL in NHL survivors not meeting public exercise guidelines is low, but can be improved with more exercise. More research on the longitudinal comparison between HL and NHL survivors and healthy controls should be performed. Lymphoma-specific questionnaires should be further developed to better understand in detail the side effects of treatment on HL and NHL survivors.

Acknowledgements This study was financially supported by the Jonker-Driessen Foundation and ZonMW - the Netherlands organization for health research and development. There were no financial disclosures.

Conflict of interest There is no conflict of interest.

Open Access This article is distributed under the terms of the Creative Commons Attribution Noncommercial License which permits any noncommercial use, distribution, and reproduction in any medium, provided the original author(s) and source are credited.

\section{References}

1. US National Coalition for Cancer Survivorship (2009) Toolbox facilitator's manual. http://www.canceradvocacy.org/. Accessed 28 Dec 2009

2. Howlader N, Noone AM, Krapcho M, Neyman N, Aminou R, Waldron W, Altekruse SF, Kosary CL, Ruhl J, Tatalovich Z, Cho
H, Mariotto A, Eisner MP, Lewis DR, Chen HS, Feuer EJ, Cronin KA, Edwards BK (2010) SEER Cancer Statistics Review, 19752008, National Cancer Institute, Bethesda, MD. http://seer.cancer. gov/csr/1975_2008/. Accessed 10 May 2011

3. Engholm G, Ferlay J, Christensen N, Bray F, Gjerstorff ML, Klint A, Kotlum JE, Olafsdottir E, Pukkala E, Storm HH (2010) NORDCANCancer Incidence, Mortality, Prevalence and Prediction in the Nordic Countries, version 3.6. Association of the Nordic Cancer Registries. Danish Cancer Society (http://www.ancr.nu). Accessed 7 Feb 2011

4. Ferlay J, Shin HR, Bray F, Forman D, Mathers C, Parkin DM (2010) Estimates of worldwide burden of cancer in 2008: GLOBOCAN 2008. Int J Canc 127(12):2893-2917

5. National Cancer Institute (2009) General information about adult non-Hodgkin's lymphoma, http://www.cancer.gov.libproxy.lib.unc. edu/cancertopics/pdq/treatment/adult-non-Hodgkins. Accessed 28 Dec 2009

6. Fobair P, Hoppe RT, Bloom J, Cox R, Varghese A, Spiegel D (1986) Psychosocial problems among survivors of Hodgkin's disease. J Clin Oncol 4(5):805-814

7. Geffen DB, Blaustein A, Amir MC, Cohen Y (2003) Posttraumatic stress disorder and quality of life in long-term survivors of Hodgkin's disease and non-Hodgkin's lymphoma in Israel. Leuk lymphoma 44(11):1925-1929

8. Knobel H, Loge J, Brit Lund M, Forfang K, Nome O, Kaasa S (2001) Late medical complications and fatigue in Hodgkin's disease survivors. J Clin Oncol 19(13):3226-3233

9. Loge JH, Abrahamsen AF, Ekeberg O, Kaasa S (1999) Reduced health-related quality of life among Hodgkin's disease survivors: a comparative study with general population norms. Ann Oncol 10 (1):71-77

10. Hess SL, Johannsdottir IM, Hamre H, Kiserud CE, Loge JH, Fossa SD (2011) Adult survivors of childhood malignant lymphoma are not aware of their risk of late effects. Acta oncologica, Stockholm

11. Mols F, Aaronson NK, Vingerhoets AJ, Coebergh JW, Vreugdenhil G, Lybeert ML, van de Poll-Franse LV (2007) Quality of life among long-term non-Hodgkin lymphoma survivors: a population-based study. Cancer 109(8):1659-1667

12. Arden-Close E, Pacey A, Eiser C (2010) Health-related quality of life in survivors of lymphoma: a systematic review and methodological critique. Leuk lymphoma 51(4):628-640

13. Mols F, Vingerhoets AJ, Coebergh JW, van de Poll-Franse LV (2005) Quality of life among long-term breast cancer survivors: a systematic review. Eur J Canc 41(17):2613-2619

14. Den Oudsten BL, Van Heck GL, De Vries J (2007) Quality of life and related concepts in Parkinson's disease: a systematic review. Mov Disord 22(11):1528-1537

15. Goodman KA, Riedel E, Serrano V, Gulati S, Moskowitz CH, Yahalom J (2008) Long-term effects of high-dose chemotherapy and radiation for relapsed and refractory Hodgkin's lymphoma. J Clin Oncol 26(32):5240-5247

16. Heutte N, Flechtner HH, Mounier N, Mellink WA, Meerwaldt JH, Eghbali $\mathrm{H}$, van't Veer MB, Noordijk EM, Kluin-Nelemans JC, Lampka E, Thomas J, Lugtenburg PJ, Viterbo L, Carde P, Hagenbeek A, van der Maazen RW, Smit WG, Brice P, van Marwijk Kooy M, Baars JW, Poortmans P, Tirelli U, Leeksma OC, Tomsic R, Feugier P, Salles G, Gabarre J, Kersten MJ, Van Den Neste E, Creemers GJ, Gaillard I, Meijnders P, Tertian G, Reman O, Muller HP, Troncy J, Blanc M, Schroyens W, Voogt PJ, Wijermans P, Rieux C, Ferme C, Henry-Amar M (2009) Quality of life after successful treatment of early-stage Hodgkin's lymphoma: 10-year follow-up of the EORTC-GELA H8 randomised controlled trial. The lancet oncology 10(12):1160-1170

17. Jerkeman M, Kaasa S, Hjermstad M, Kvaloy S, Cavallin-Stahl E (2001) Health-related quality of life and its potential prognostic implications in patients with aggressive lymphoma: a Nordic Lymphoma Group Trial. Med oncol 18(1):85-94 
18. Osoba D, Rodrigues G, Myles J, Zee B, Pater J (1998) Interpreting the significance of changes in health-related qualityof-life scores. J Clin Oncol 16(1):139-144

19. Ware JE, Kosinski MA (2001) SF-36 ${ }^{\circledR}$ physical and mental health summary scales: a manual for users of version 1, 2nd edn. QualityMetric Inc, Lincoln

20. Ware JE, Kosinski MA, Dewey JE (2000) How to score version 2 of the SF-36 health survey. Quality Metric Inc., Lincoln

21. Norman GR, Sloan JA, Wyrwich KW (2003) Interpretation of changes in health-related quality of life: the remarkable universality of half a standard deviation. Medical care 41(5):582-592

22. Brandt J, Dietrich S, Meissner J, Neben K, Ho AD, Witzens-Harig M (2010) Quality of life of long-term survivors with Hodgkin lymphoma after high-dose chemotherapy, autologous stem cell transplantation, and conventional chemotherapy. Leuk lymphoma 51(11):2012-2020

23. Ganz PA, Moinpour CM, Pauler DK, Kornblith AB, Gaynor ER, Balcerzak SP, Gatti GS, Erba HP, McCoy S, Press OW, Fisher RI (2003) Health status and quality of life in patients with early-stage Hodgkin's disease treated on Southwest Oncology Group Study 9133. J Clin Oncol 21(18):3512-3519

24. Gil-Fernandez J, Ramos C, Tamayo T, Tomas F, Figuera A, Arranz R, Martinez-Chamorro C, Fernandez-Ranada M (2003) Quality of life and psychological well-being in Spanish long-term survivors of Hodgkin's disease: results of a controlled pilot study. Ann Hematol 82(1):14-18

25. Greil R, Holzner B, Kemmler G, Kopp M, Buchowski A, Oberaigner W, Fritsch E, Dirnhofer S, Rueffer U, Diehl V, Sperner-Unterweger B (1999) Retrospective assessment of quality of life and treatment outcome in patients with Hodgkin's disease from 1969 to 1994. Eur J Cancer 35(5):698-706

26. Hjermstad MJ, Oldervoll L, Fossa SD, Holte H, Jacobsen AB, Loge JH (2006) Quality of life in long-term Hodgkin's disease survivors with chronic fatigue. Eur J Cancer 42(3):327-333

27. Joly F, Henry-Amar M, Arveux P, Reman O, Tanguy A, Peny AM, Lebailly P, Mace-Lesec'h J, Vie B, Genot JY, Busson A, Troussard X, Leporrier M (1996) Late psychosocial sequelae in Hodgkin's disease survivors: a French population-based case-control study. J Clin Oncol 14(9):2444-2453

28. Mols F, Vingerhoets AJ, Coebergh JW, Vreugdenhil G, Aaronson NK, Lybeert ML, van de Poll-Franse LV (2006) Better quality of life among 10-15 year survivors of Hodgkin's lymphoma compared to 5-9 year survivors: a population-based study. Eur J Cancer 42(16):2794-2801

29. Norum J, Wist EA (1996) Quality of life in survivors of Hodgkin's disease. Qual Life Res 5(3):367-374

30. van Tulder MW, Aaronson NK, Bruning PF (1994) The quality of life of long-term survivors of Hodgkin's disease. Ann Oncol 5(2):153-158

31. Wettergren L, Bjorkholm M, Axdorph U, Bowling A, Langius-Eklof A (2003) Individual quality of life in long-term survivors of Hodgkin's lymphoma-a comparative study. Qual Life Res 12(5):545-554

32. Wettergren L, Bjorkholm M, Axdorph U, Langius-Eklof A (2004) Determinants of health-related quality of life in long-term survivors of Hodgkin's lymphoma. Qual Life Res 13(8):1369-1379

33. Bellizzi KM, Rowland JH, Arora NK, Hamilton AS, Miller MF, Aziz NM (2009) Physical activity and quality of life in adult survivors of non-Hodgkin's lymphoma. J Clin Oncol 27(6):960-966
34. Doorduijn J, Buijt I, Holt B, Steijaert M, Uyl-de Groot C, Sonneveld P (2005) Self-reported quality of life in elderly patients with aggressive non-Hodgkin's lymphoma treated with CHOP chemotherapy. Eur J Haematol 75(2):116-123

35. Merli F, Bertini M, Luminari S, Mozzana R, Berte R, Trottini M, Stelitano C, Botto B, Pizzuti M, Quintana G, De Paoli A, Federico M (2004) Quality of life assessment in elderly patients with aggressive non-Hodgkin's lymphoma treated with anthracyclinecontaining regimens. Report of a prospective study by the Intergruppo Italiano Linfomi. Haematologica 89(8):973-978

36. Pettengell R, Donatti C, Hoskin P, Poynton C, Kettle PJ, Hancock B, Johnson S, Dyer MJ, Rule S, Walker M, Wild D (2008) The impact of follicular lymphoma on health-related quality of life. Ann Oncol 19(3):570-576

37. Reeve BB, Potosky AL, Smith AW, Han PK, Hays RD, Davis WW, Arora NK, Haffer SC, Clauser SB (2009) Impact of cancer on health-related quality of life of older Americans. J Natl Canc Instit 101(12):860-868

38. Smith SK, Crespi CM, Petersen L, Zimmerman S, Ganz PA (2010) The impact of cancer and quality of life for post-treatment non-Hodgkin lymphoma survivors. Psychooncology 19 (12):1259-1267

39. Smith SK, Zimmerman S, Williams CS, Zebrack BJ (2009) Health status and quality of life among non-Hodgkin lymphoma survivors. Cancer 115(14):3312-3323

40. Vallance JK, Courneya KS, Jones LW, Reiman T (2005) Differences in quality of life between non-Hodgkin's lymphoma survivors meeting and not meeting public health exercise guidelines. Psycho-Oncology 14(11):979-991

41. Aaronson NK, Ahmedzai S, Bergman B, Bullinger M, Cull A, Duez NJ, Filiberti A, Flechtner H, Fleishman SB, de Haes JC et al (1993) The European Organization for Research and Treatment of Cancer QLQ-C30: a quality-of-life instrument for use in international clinical trials in oncology. J Natl Canc Instit 85 (5):365-376

42. Ware JE Jr, Sherbourne CD (1992) The MOS 36-item short-form health survey (SF-36). I. Conceptual framework and item selection. Med care 30(6):473-483

43. Browne JP, O'Boyle CA, McGee HM, McDonald NJ, Joyce CR (1997) Development of a direct weighting procedure for quality of life domains. Qual Life Res 6(4):301-309

44. Cella DF, Tulsky DS, Gray G, Sarafian B, Linn E, Bonomi A, Silberman M, Yellen SB, Winicour P, Brannon J et al (1993) The functional assessment of cancer therapy scale: development and validation of the general measure. J Clin Oncol 11 (3): $570-579$

45. Shipp M, Harrington D, Anderson J (1993) A predictive model for aggressive non-Hodgkin's lymphoma. The international nonhodgkin's lymphoma prognostic factors project. New Engl J Med 329(14):987-994

46. van de Poll-Franse LV, Mols F, Gundy CM, Creutzberg CL, Nout RA, Verdonck-de Leeuw IM, Taphoorn MJ, Aaronson NK (2010) Normative data for the EORTC QLQ-C30 and EORTC-sexuality items in the general Dutch population. Eur J Cancer 47(5):667675

47. American cancer society (2009) Cancer facts and figures 2009. American Cancer Society, Atlanta 